\title{
Lowering of Proteinuria in Response to Antihypertensive Therapy Predicts Improved Renal Function in Late but Not in Early Diabetic Nephropathy: A Pooled Analysis
}

\author{
George Jerums $^{\mathrm{a}, \mathrm{b}}$ Sianna Panagiotopoulos ${ }^{\mathrm{a}, \mathrm{b}} \quad$ Erosha Premaratne $^{\mathrm{a}, \mathrm{b}}$ \\ David A. Power ${ }^{b, c}$ Richard J. Maclsaac ${ }^{a, b}$ \\ ${ }^{a}$ Endocrine Centre and Departments of ${ }^{b}$ Medicine and ${ }^{\mathrm{C}}$ Nephrology, Austin Health and University of Melbourne, \\ Heidelberg, Vic., Australia
}

\section{Key Words \\ Hypertension - Microalbuminuria - Albuminuria • Glomerular filtration rate $\cdot$ Diabetic nephropathy}

\begin{abstract}
In late diabetic nephropathy (DN) the initial lowering of albumin excretion rate (AER) with antihypertensive therapy is proportional to the degree of subsequent preservation of glomerular filtration rate (GFR). Whether a similar relationship exists between AER and GFR in early diabetes is not known. The present analysis has compared AER and GFR responses to antihypertensive therapy in 33 published studies (77 treatment groups) of early and late DN in type 1 (T1) and type 2 (T2) diabetes, analyzed on an intention-to-treat basis. Prospective trials were included if the initial change in AER during the first year of therapy and the change in GFR during at least 2 years of follow-up could be estimated from group mean data. The initial \% decreases in AER were $5.9 \pm 4.3$ (T1), $10.5 \pm 5.4(\mathrm{~T} 2$, normotensive) and $18.4 \pm 6.2$ ( $\mathrm{T} 2$, hypertensive) in early DN and $7.6 \pm 11.1$ (T1) and $20.8 \pm 5.5$ (T2) in late DN. The corresponding annual \% rates of decline of GFR were $2.0 \pm 0.5(\mathrm{~T} 1), 1.6 \pm 0.5$ ( $\mathrm{T} 2$, normotensive) and $2.1 \pm$ 0.3 (T2, hypertensive) in early DN and $9.8 \pm 1.5(\mathrm{~T} 1)$ and 9.2 \pm 1.1 (T2) in late DN. AER and GFR responses in each treat-
\end{abstract}

\section{KARGER}

Fax +41613061234

E-Mail karger@karger.ch

www.karger.com (c) 2008 S. Karger AG, Basel

0250-8095/08/0284-0614\$24.50/0

Accessible online at:

www.karger.com/ajn ment group were closely correlated in late nephropathy $(\mathrm{T} 1$, $r=-0.67, p=0.03 ; T 2, r=0.57, p=0.02$ ) but not in early nephropathy. In contrast to late $D N$, the initial decrease in AER with antihypertensive therapy was not shown to predict the subsequent rate of decline of GFR in early DN. It follows that assessment of renoprotection during antihypertensive therapy in early nephropathy should be based not only on albuminuria but also on the GFR response.

Copyright $\odot 2008$ S. Karger AG, Basel

\section{Introduction}

Hypertension is both a cause and effect of renal impairment and its treatment influences renal outcomes. A meta-analysis of several large intervention studies has shown that a lower mean blood pressure during therapy is associated with slower rates of decline of glomerular filtration rate (GFR) [1]. However, attained blood pressure is not the only determinant of the rate of progression of renal impairment. For instance, the level of proteinuria and the reduction of proteinuria during antihypertensive therapy may also influence the rate of decline of renal function. An early reduction in proteinuria associated with hypertensive therapy was first shown to predict the 
long-term decline in GFR, using within patient comparisons, in two small studies of overt nephropathy in diabetic [2] and non-diabetic kidney disease [3, 4].

Subsequent studies of overt diabetic nephropathy using group mean data also support the concept that the early antiproteinuric response to antihypertensive therapy predicts the subsequent decline in renal function. In patients with type 1 diabetes and overt nephropathy, the Collaborative Study Group showed that captopril therapy, supplemented as necessary with other antihypertensive agents, resulted in a decrease in proteinuria of around $40 \%$ and an approximate halving of the rate of doubling of serum creatinine [5]. In patients with type 2 diabetes and late nephropathy, two large studies of angiotensin receptor blockade examined the independent role of proteinuria and changes in proteinuria as predictors of the subsequent rate of decline of renal function.

In the Reduction of Endpoints in NIDDM with the Angiotensin II Antagonist Losartan (RENAAL) study [6], changes in albuminuria in the first 6 months of therapy with losartan were related to the degree of long-term renoprotection. A reduction in albuminuria of around $50 \%$ was associated with a $36 \%$ reduction in the risk of doubling of serum creatinine, ESRD or death [7]. This renoprotective effect was still demonstrable after controlling for the level of attained blood pressure. In the Irbesartan Diabetic Nephropathy Trial (IDNT) [8], for each halving of proteinuria during the first 12 months of therapy with irbesartan, amlodipine or placebo, the risk for renal failure was reduced by 56\% [9]. Furthermore, multivariate analysis showed that proteinuria reduction in the first 12 months of irbesartan therapy accounted for $36 \%$ of the total renoprotective effect observed.

In early diabetic nephropathy (microalbuminuria), studies of antihypertensive therapy with ACE inhibitors have shown reductions in albumin excretion rate (AER) of $>50 \%$ which are comparable in percentage terms to those reported in overt nephropathy. In hypertensive patients with type 1 diabetes and early nephropathy there was a $70 \%$ reduction of albuminuria during captopril therapy but no significant change in GFR was noted during 2 years of follow-up [10]. Similarly, a study of hypertensive patients with type 2 diabetes and early nephropathy showed a $70 \%$ reduction in albuminuria during treatment with irbesartan at a dose of $300 \mathrm{mg}$ daily but no significant change in renal function over 2 years [11].

The above studies raise the question of whether the initial antialbuminuric response to antihypertensive therapy in early diabetic kidney disease can predict the rate of decline in GFR previously shown in studies of overt nephropathy. The present analysis has therefore examined the degree of coupling of the albuminuric and GFR responses to antihypertensive therapy, using group mean data, on an intention-to-treat basis, in studies of at least 2 years duration in patients with early and late diabetic nephropathy.

\section{Methods}

Studies of antihypertensive therapy in early and late diabetic nephropathy were considered for inclusion in the analysis based on a Medline literature search (1966-September 2006) using the search strategy described by Strippoli et al. [12]. The present analysis was based on all references quoted in a meta-analysis of antihypertensive therapy in diabetes by Strippoli et al. A search for additional studies using references in papers quoted by Strippoli et al. was also performed. The criteria for inclusion of a study in the analysis were: (1) Prospective study of antihypertensive therapy in diabetic patients with at least 2 years of follow-up. (2) Inclusion of a parallel or sequential comparator group. (3) Sufficient data to allow estimation of the AER response as a percentage of baseline in the first year of follow-up. (4) Sufficient data to allow estimation of the annual rate of decline of GFR during the entire study.

All studies meeting these criteria were included in the analysis.

In the systematic review of antihypertensive therapy and diabetic nephropathy by Strippoli et al. [12], 24 of 59 studies met the above criteria. In addition, nine other studies were included. Inclusion was based on two main criteria. Firstly, if the change in albuminuria (AER or albumin creatinine ratio) or proteinuria during the first 12 months of therapy could be estimated from mean data for each treatment group on an intention-to-treat basis. Secondly, if the change in GFR during at least 2 years of follow-up could be estimated from group mean data.

All albuminuria data were expressed as $\mu \mathrm{g} / \mathrm{min}$, using the following equivalents: $200 \mu \mathrm{g} / \mathrm{min}=300 \mathrm{mg} / 24 \mathrm{~h}=$ total proteinuria $500 \mathrm{mg} / 24 \mathrm{~h}=300 \mathrm{mg}$ albumin per gram of creatinine [13]. The initial AER response was defined as percentage change in AER during the first 12 months of therapy. Hypertension was defined as resting blood pressure $>140 / 90 \mathrm{~mm} \mathrm{Hg}$.

For the purposes of this analysis, each study was categorized according to group mean data for baseline albuminuria and final GFR. In studies of type 1 diabetes, early diabetic nephropathy was defined as baseline AER between 30 and $300 \mathrm{mg} / 24 \mathrm{~h}$ and final GFR $>90 \mathrm{ml} / \mathrm{min}$ or $>90 \mathrm{ml} / \mathrm{min} / 1.73 \mathrm{~m}^{2}$. All 'microalbuminuria' studies were classified as early diabetic nephropathy, equivalent to CKD stage 1 . The onset of the clinical phase of diabetic nephropathy is signalled, by convention, by the appearance of persistent proteinuria (total daily protein excretion of $0.5 \mathrm{~g}$ or more), which corresponds to an AER greater than about $200 \mu \mathrm{g} / \mathrm{min}$ (i.e. about $300 \mathrm{mg} /$ day) [13]. Late diabetic nephropathy was therefore defined as baseline albuminuria $>300 \mathrm{mg} / 24 \mathrm{~h}$ or total proteinuria $>500 \mathrm{mg} / 24 \mathrm{~h}$ and final GFR $\leq 90 \mathrm{ml} / \mathrm{min}$ or $\leq 90 \mathrm{ml} /$ $\min / 1.73 \mathrm{~m}^{2}$. All 'macroalbuminuria' studies were classified as late nephropathy, equivalent to CKD stage 2 or 3 . One study in normotensive patients with type 1 diabetes, macroalbuminuria 
and final GFR $>90 \mathrm{ml} / \mathrm{min}$ [14] could not be classified and was therefore excluded from analysis.

In studies of type 2 diabetes, early and late nephropathy were defined according to baseline albuminuria as for type 1 diabetes and final GFR greater or less than $75 \mathrm{ml} / \mathrm{min}$ or $75 \mathrm{ml} / \mathrm{min} / 1.73$ $\mathrm{m}^{2}$, respectively. The use of a lower GFR cut-off was based on the age-related decline in GFR in subjects with type 2 diabetes [15].

All microalbuminuria studies in type 2 diabetes were classified as early nephropathy, equivalent to CKD stage 1 or early stage 2 . All macroalbuminuria studies in type 2 diabetes were classified as late nephropathy, equivalent to CKD late stage 2 or stage 3 . One study in patients with type 2 diabetes, microalbuminuria and final GFR $<75 \mathrm{ml} / \mathrm{min} / 1.73 \mathrm{~m}^{2}$ [16] and one study in patients with type 2 diabetes, macroalbuminuria and final GFR $>75 \mathrm{ml} / \mathrm{min}$ [17] could not be classified and both were therefore excluded from analysis.

In all early nephropathy studies involving patients with type 1 diabetes $(n=9)$, proteinuria was assessed by AER. In studies of late nephropathy involving patients with type 1 diabetes $(n=5)$, proteinuria was assessed by AER in four studies and total proteinuria $>0.5 \mathrm{~g} / 24 \mathrm{~h}$ in one study. All studies of early nephropathy in normotensive $(n=5)$ or hypertensive $(n=8)$ patients with type 2 diabetes used AER to assess albuminuria. In studies of late nephropathy $(n=7)$ involving patients with type 2 diabetes, proteinuria was assessed by AER in three studies, albumin to creatinine ratio $>30(\mathrm{mg} / \mathrm{g})$ in one study and total proteinuria $>500 \mathrm{mg} /$ $24 \mathrm{~h}$ in three studies. The initial response of proteinuria to antihypertensive therapy was calculated as the percentage change in proteinuria in the first 3-12 months using group mean data.

GFR was measured by isotopic methods in 14 studies, by iohexol in three studies, by inulin in one study and by creatinine clearance in 12 studies. GFR was estimated by the CockcroftGault formula in two studies, and by the simplified four variable modification of diet in renal disease (MDRD) formula in one study. The annual percentage change in measured or estimated GFR was calculated from group mean data over $2-8$ years using baseline GFR and the last value on therapy. To allow for variations in methods of reporting proteinuria and renal function in different studies, group mean changes in these parameters were calculated as a percentage of baseline values. Three large studies of late nephropathy $[5,6,8]$ reported changes in total proteinuria and creatinine clearance or creatinine-based estimate of GFR. Percentage changes in these parameters were in agreement with other studies of late nephropathy, which used albuminuria and direct GFR measurements. In this analysis, the terms AER and GFR are therefore used in a generic sense to refer to renal endpoints in both early and late nephropathy. Initial \% changes in AER and annual $\%$ changes in GFR were calculated in three categories of early nephropathy studies (type 1 , type 2 normotensive, type 2 hypertensive) and two categories of late nephropathy studies (type 1 and type 2). Because the presence of hypertension influences both the design of intervention studies as well as the progression of nephropathy, studies in hypertensive patients with type 2 diabetes and early nephropathy were analyzed separately from those in normotensive patients.

The relationship between percentage changes in AER and GFR to mean arterial blood pressure (diastolic blood pressure and one third of pulse pressure) was also assessed. Changes in AER and GFR in each treatment group were related to final mean arterial pressure and to change in mean arterial pressure during the study.

\section{Results}

The effects of antihypertensive therapy were analyzed in 33 studies. Thirty-one studies were prospective, randomized studies of ACE inhibition or angiotensin receptor blockade with concurrent control groups allocated to other antihypertensive agents to minimize differences in attained blood pressure. Two early studies were within group studies before and during 'conventional' antihypertensive therapy $[18,19]$.

Table 1 summarizes the distribution of treatment groups according to diabetes type, stage of nephropathy and nature of intervention. An inhibitor of the renin-angiotensin system (RAS) was used in 31 of 77 treatment groups, with the majority being ACE inhibitors. The details of the 33 studies in the analysis are summarized according to study category in table $2 \mathrm{a}-\mathrm{e}$.

There were more early than late nephropathy studies in this analysis, but the three largest studies $[5,6,8]$ were performed in late nephropathy (table 2). Mean study duration was comparable in early $(4.0 \pm 0.4$ years $)$ and late nephropathy studies $(3.2 \pm 0.4$ years, $\mathrm{p}=0.15)$.

Baseline albuminuria and GFR in the five study categories are shown in figure 1. There is clear separation of early and late studies according to baseline AER (fig. 1a) but there is overlap in baseline GFR (fig. 1b). This is the basis for the classification of stage of nephropathy according to final rather than baseline GFR.

The initial percentage changes in AER and the overall percentage changes in GFR are presented in five categories according to diabetes type and stage of nephropathy, as shown in figure 2 . In type 1 diabetes studies, the initial decrease in albuminuria was $5.9 \pm 4.3 \%$ in early and 7.6 $\pm 11.1 \%$ in late nephropathy $(\mathrm{p}=0.38)$. In type 2 diabetes studies, the initial decrease in albuminuria was similar in hypertensive and in normotensive early nephropathy $(18.4 \pm 6.2 \%$ vs. $10.5 \pm 5.4 \%, \mathrm{p}=0.38)$. The initial decrease in albuminuria in late type 2 nephropathy was 20.8 $\pm 5.5 \%$ ( $p=0.21$ vs. early normotensive, $\mathrm{p}=0.78$ vs. early hypertensive).

The rates of annual decline in GFR expressed as $\mathrm{ml} /$ $\mathrm{min}$ or $\mathrm{ml} / \mathrm{min} / 1.73 \mathrm{~m}^{2}$ for the five study groups according to diabetes type, presence or absence of hypertension and early or late stage nephropathy are shown in table 3. Because of differences in baseline renal function, rates of decline in GFR in early and late nephropathy were expressed in absolute terms before comparisons were made. In both type 1 and type 2 diabetes, the absolute rate of decline of GFR was greater in late than in early nephropathy studies. 
Table 1. Classification of study groups according to type of antihypertensive therapy

\begin{tabular}{|c|c|c|c|c|c|c|c|}
\hline Intervention & $\begin{array}{l}\text { Type } 1 \\
\text { early }\end{array}$ & $\begin{array}{l}\text { Type } 1 \\
\text { late }\end{array}$ & $\begin{array}{l}\text { Type } 2 \\
\text { early NT }\end{array}$ & $\begin{array}{l}\text { Type } 2 \\
\text { early HT }\end{array}$ & $\begin{array}{l}\text { Type } 2 \\
\text { late }\end{array}$ & $\begin{array}{l}\text { Treatment } \\
\text { groups }\end{array}$ & Subjects \\
\hline $\mathrm{ACE}_{\mathrm{i}}$ & 11 & 3 & 4 & 5 & 4 & 27 & $1,109^{1}$ \\
\hline $\mathrm{ARB}$ & & & & 2 & 2 & 4 & 1,719 \\
\hline СCB & 3 & & 1 & 2 & 3 & 9 & $935^{1}$ \\
\hline Standard (incl. BB, diuretic) & 0 & 3 & 0 & 1 & 3 & 7 & 98 \\
\hline Standard + placebo & & 1 & & 3 & 2 & 6 & 1,767 \\
\hline Placebo only & 8 & & 4 & & & 12 & 397 \\
\hline Untreated & 1 & 3 & & & & 4 & 51 \\
\hline Moderate & & & 1 & 2 & 1 & 4 & $416^{1}$ \\
\hline Intensive & & & 1 & 2 & 1 & 4 & $413^{1}$ \\
\hline Treatment groups (total $=77$ ) & 23 & 10 & 11 & 17 & 16 & & \\
\hline Studies $($ total $=33)$ & 9 & 5 & 5 & 7 & 7 & & \\
\hline Subjects & 681 & 510 & 407 & 1,804 & 3,503 & & $6,905^{1}$ \\
\hline
\end{tabular}

${ }^{1} \mathrm{ABCD}$ study ( $\mathrm{n}=470$ patients) shown twice.

$\mathrm{ACE}_{\mathrm{i}}=\mathrm{ACE}$ inhibitor; $\mathrm{ARB}=$ angiotensin receptor blocker; $\mathrm{CCB}=$ calcium channel blocker; Standard $=$ non- $\mathrm{ACE}$, non- $\mathrm{ARB}$, non-CCB including $\beta$-blockers (BB) and diuretics; Moderate, intensive = levels of blood pressure control as defined for early nephropathy in the ABCD trial [40] and for late nephropathy by Joss et al. [43].

Table 2a. Normotensive type 1, early DN

\begin{tabular}{|c|c|c|c|c|c|c|c|c|c|c|c|c|c|}
\hline \multirow[t]{2}{*}{ Author } & \multirow{2}{*}{$\begin{array}{l}\text { Study } \\
\text { duration } \\
\text { years }\end{array}$} & \multicolumn{8}{|c|}{ Intervention } & \multirow{2}{*}{$\begin{array}{l}\text { GFR } \\
\text { method }\end{array}$} & \multirow[t]{2}{*}{ PP } & \multicolumn{2}{|c|}{ Mean baseline GFR } \\
\hline & & $\begin{array}{l}\Delta \text { AER } \\
\%\end{array}$ & $\mathrm{n}$ & $\begin{array}{l}\Delta \text { GFR } \\
\%\end{array}$ & $\begin{array}{l}\Delta \text { AER } \\
\%\end{array}$ & $\mathrm{n}$ & $\begin{array}{l}\Delta \text { GFR } \\
\%\end{array}$ & $\begin{array}{l}\Delta \text { AER n } \\
\%\end{array}$ & $\begin{array}{l}\Delta \mathrm{GFR} \\
\%\end{array}$ & & & $\begin{array}{l}\mathrm{ml} / \mathrm{min} / \\
1.73 \mathrm{~m}^{2}\end{array}$ & $\begin{array}{l}\mathrm{ml} / \\
\mathrm{min}\end{array}$ \\
\hline $\begin{array}{l}\text { Mathiesen } \\
1991 \text { [44] } \\
1999 \text { [45] }\end{array}$ & 8 & 12 & $\begin{array}{c}\text { untre } \\
23\end{array}$ & ed & -2.1 & $\begin{array}{c}\text { capto } \\
21\end{array}$ & il -1.1 & & & ${ }^{51} \mathrm{Cr}-\mathrm{EDTA}$ & AER & & 128 \\
\hline $\begin{array}{l}\text { Hansen } \\
1994[46]\end{array}$ & 2 & \multicolumn{3}{|c|}{ placebo } & \multicolumn{3}{|c|}{ captopril } & & & $\begin{array}{l}{ }^{125} \text { I-iotha- } \\
\text { lamate }\end{array}$ & AER & 133 & \\
\hline $\begin{array}{l}\text { Microalbumi- } \\
\text { nuria Captopri } \\
\text { Study Group } \\
1996[10]\end{array}$ & $\mathrm{il}^{2}$ & 2 & $\begin{array}{l}\text { place } \\
114\end{array}$ & -6.7 & -30 & $\begin{array}{c}\text { capto } \\
111\end{array}$ & il -1.5 & & & $\mathrm{CC}$ & AER & 95 & \\
\hline $\begin{array}{l}\text { Crepaldi } \\
1998 \text { [47] }\end{array}$ & 3 & 11.7 & placebo & -1.5 & -15.4 & lisinopril & il -1.2 & \multicolumn{2}{|c|}{$\begin{array}{l}\text { nifedipine } \\
26\end{array}$} & ${ }^{51} \mathrm{Cr}-\mathrm{EDTA}$ & AER & 116 & \\
\hline $\begin{array}{l}\text { Atlantis } \\
2000[48]\end{array}$ & 2 & 0 & $\begin{array}{c}\text { place } \\
46\end{array}$ & 1.75 & $-20^{\mathrm{ram}}$ & $\begin{array}{r}\text { pril ( } \\
44\end{array}$ & $\begin{array}{l}25 \mathrm{mg}) \\
-0.7\end{array}$ & $-36 \quad 4$ & $\begin{array}{l}5 \mathrm{mg}) \\
-3.4\end{array}$ & iohexol & AER & & 104 \\
\hline $\begin{array}{l}\text { Esprit } \\
2001[49]\end{array}$ & 3 & -45 & $\begin{array}{c}\text { enala } \\
18\end{array}$ & il -4.1 & 25 & $\begin{array}{c}\text { nifedi } \\
18\end{array}$ & -4.1 & $\begin{array}{cc} & \mathrm{pl}_{c} \\
0 & 1\end{array}$ & -4.1 & iohexol & AER & 103 & \\
\hline $\begin{array}{l}\text { Jerums } \\
2001[50]\end{array}$ & 3 & -45 & $\begin{array}{c}\text { erind } \\
13\end{array}$ & bril & 17.5 & $\begin{array}{c}\text { hifedi } \\
10\end{array}$ & ne 7.4 & $27.6 \frac{\mathrm{pl}_{\mathrm{c}}}{1}$ & o & 99 mTc-DTPA & AER & 103 & \\
\hline $\begin{array}{l}\text { Bojestig } \\
2001[51]\end{array}$ & 2 & \multicolumn{2}{|c|}{ placebo } & -2.8 & $\begin{array}{l}\mathrm{ram} \\
0.9\end{array}$ & $\begin{array}{r}\text { pril ( } \\
19\end{array}$ & $\begin{array}{l}25 \mathrm{mg}) \\
-2.5\end{array}$ & $-13^{\text {ramip }}$ & $\begin{array}{l}5 \mathrm{mg}) \\
2.0\end{array}$ & iohexol & AER & 103 & \\
\hline $\begin{array}{l}\text { Poulsen } \\
2001 \text { [52] }\end{array}$ & 2 & 10 & $\begin{array}{l}\text { place } \\
10\end{array}$ & 1.2 & -23 & $\begin{array}{r}\text { lisino } \\
12\end{array}$ & 2.2 & & & $\begin{array}{l}{ }^{125} \text { I-iotha- } \\
\text { lamate }\end{array}$ & AER & 133 & \\
\hline
\end{tabular}

$\mathrm{PP}=$ Proteinuria parameter $\mathrm{CC}=$ creatinine clearance; $\mathrm{n}=$ number of patients; $\Delta$ AER $\%=$ initial percentage change in AER or equivalent during first year of study; $\Delta$ GFR $\%$ = annual percentage change in GFR or equivalent assessed over entire study period. 
Table 2b. Normotensive type 2, early DN

\begin{tabular}{|c|c|c|c|c|c|c|c|c|c|c|c|c|c|}
\hline \multirow[t]{2}{*}{ Author } & \multirow{2}{*}{$\begin{array}{l}\text { Study } \\
\text { duration } \\
\text { years }\end{array}$} & \multicolumn{8}{|c|}{ Intervention } & \multirow{2}{*}{$\begin{array}{l}\text { GFR } \\
\text { method }\end{array}$} & \multirow[t]{2}{*}{ PP } & \multicolumn{2}{|c|}{ Mean baseline GFR } \\
\hline & & $\begin{array}{l}\Delta \text { AER } \\
\%\end{array}$ & $\mathrm{n}$ & $\begin{array}{l}\Delta \text { GFR } \\
\%\end{array}$ & $\begin{array}{l}\Delta \text { AER } \\
\%\end{array}$ & $\mathrm{n}$ & $\begin{array}{l}\Delta \text { GFR } \\
\%\end{array}$ & $\begin{array}{l}\Delta \text { AER n } \\
\%\end{array}$ & $\begin{array}{l}\Delta \text { GFR } \\
\%\end{array}$ & & & $\begin{array}{l}\mathrm{ml} / \mathrm{min} / \\
1.73 \mathrm{~m}^{2}\end{array}$ & $\begin{array}{l}\mathrm{ml} / \\
\mathrm{min}\end{array}$ \\
\hline Sano & 4 & \multicolumn{3}{|c|}{ placebo } & \multicolumn{3}{|c|}{ enalapril } & & & \multirow[t]{2}{*}{ CC } & \multirow[t]{2}{*}{ AER } & & \multirow[t]{2}{*}{91} \\
\hline $1994[53]$ & & 6.1 & 12 & -0.9 & -29.4 & 12 & 1.3 & & & & & & \\
\hline Ahmad & 5 & \multicolumn{3}{|c|}{ placebo } & \multicolumn{3}{|c|}{ enalapril } & & & \multirow[t]{2}{*}{ insulin } & \multirow[t]{2}{*}{ AER } & \multirow[t]{2}{*}{124} & \\
\hline $1997[54]$ & & 0 & 51 & -0.8 & -12.7 & 52 & -0.8 & & & & & & \\
\hline Ravid & 6 & \multicolumn{3}{|c|}{ placebo } & \multicolumn{3}{|c|}{ enalapril } & & & \multirow[t]{2}{*}{$\mathrm{CC}$} & \multirow[t]{2}{*}{ AER } & \multirow[t]{2}{*}{108} & \\
\hline $1998[55]$ & & 8.9 & 79 & -2.6 & -14.7 & 77 & -0.2 & & & & & & \\
\hline Schrier & 5 & \multicolumn{3}{|c|}{ moderate } & \multicolumn{3}{|c|}{ intensive } & & & \multirow[t]{3}{*}{$\mathrm{CC}$} & \multirow[t]{3}{*}{ AER } & \multirow[t]{3}{*}{85} & \\
\hline $2002[42]$ & & 0 & 60 & -3.3 & -26 & 49 & -2.3 & & & & & & \\
\hline ABCD & & & & & & & & & & & & & \\
\hline Jerums & 6 & \multicolumn{3}{|c|}{ perindopril } & \multicolumn{3}{|c|}{ nifedipine } & \multicolumn{2}{|c|}{ placebo } & $99 \mathrm{~m}$ Tc-DTPA & AER & 92 & \\
\hline $2004[32]$ & & -4.75 & 23 & -2.6 & -9 & 27 & -1.5 & 27 & -4.3 & & & & \\
\hline
\end{tabular}

$\mathrm{PP}=$ Proteinuria parameter; $\mathrm{CC}=$ creatinine clearance; $\mathrm{n}=$ number of patients; $\Delta \mathrm{AER} \%=$ initial percentage change in AER or equivalent during first year of study; $\Delta$ GFR $\%=$ annual percentage change in GFR or equivalent assessed over entire study period.

Table 2c. Hypertensive type 2, early DN

\begin{tabular}{|c|c|c|c|c|c|c|c|c|c|c|c|c|c|}
\hline \multirow[t]{2}{*}{ Author } & \multirow{2}{*}{$\begin{array}{l}\text { Study } \\
\text { duration } \\
\text { years }\end{array}$} & \multicolumn{8}{|c|}{ Intervention } & \multirow{2}{*}{$\begin{array}{l}\text { GFR } \\
\text { method }\end{array}$} & \multirow[t]{2}{*}{$\mathrm{PP}$} & \multicolumn{2}{|c|}{ Mean baseline GFR } \\
\hline & & $\begin{array}{l}\Delta \text { AER } \\
\%\end{array}$ & $\mathrm{n}$ & $\begin{array}{l}\Delta \text { GFR } \\
\%\end{array}$ & $\begin{array}{l}\Delta \text { AER } \\
\%\end{array}$ & $\mathrm{n}$ & $\begin{array}{l}\Delta \text { GFR } \\
\%\end{array}$ & $\begin{array}{l}\Delta \text { AER } \quad n \\
\%\end{array}$ & $\begin{array}{l}\Delta \text { GFR } \\
\%\end{array}$ & & & $\begin{array}{l}\mathrm{ml} / \mathrm{min} / \\
1.73 \mathrm{~m}^{2}\end{array}$ & $\begin{array}{l}\mathrm{ml} / \\
\mathrm{min}\end{array}$ \\
\hline Lacourciere & 3 & \multicolumn{3}{|c|}{ captopril \pm HCT } & \multicolumn{3}{|c|}{ metoprolol \pm HCT } & & & \multirow[t]{2}{*}{${ }^{51} \mathrm{Cr}$-EDTA } & \multirow[t]{2}{*}{ AER } & \multirow[t]{2}{*}{93} & \\
\hline $1993[56]$ & & -77 & & -2.10 & -46 & 12 & -0.4 & & & & & & \\
\hline Sano & 4 & \multicolumn{3}{|c|}{ placebo } & \multicolumn{3}{|c|}{ enalapril } & & & \multirow[t]{2}{*}{ CC } & \multirow[t]{2}{*}{ AER } & & \multirow[t]{2}{*}{90} \\
\hline $1994[53]$ & & 14.6 & 13 & -0.8 & -16.7 & 11 & 1.2 & & & & & & \\
\hline Chan & 5.5 & \multicolumn{3}{|c|}{ enalapril } & \multicolumn{3}{|c|}{ nifedipine } & & & \multirow[t]{2}{*}{ CC } & \multirow[t]{2}{*}{ AER } & & \multirow[t]{2}{*}{75} \\
\hline $2000[57]$ & & -54 & 50 & -3.5 & 11 & 52 & -3.4 & & & & & & \\
\hline Parving & 2 & \multicolumn{3}{|c|}{ placebo } & \multicolumn{3}{|c|}{ irbesartan (150 mg) } & \multicolumn{2}{|c|}{ irbesartan (300 mg) } & \multirow[t]{2}{*}{ CC } & \multirow[t]{2}{*}{ AER } & \multirow[t]{2}{*}{109} & \\
\hline (RMA-2 & & -2 & 201 & -2.4 & -2.4 & 195 & -2.7 & $\begin{array}{ll}-38 & 19\end{array}$ & -3.2 & & & & \\
\hline Estacio & 5 & \multicolumn{3}{|c|}{ moderate } & \multicolumn{3}{|c|}{ intensive } & & & \multirow[t]{2}{*}{$\mathrm{CC}$} & \multirow[t]{2}{*}{ AER } & & \multirow[t]{2}{*}{$85^{1}$} \\
\hline $\begin{array}{l}2000[58] \\
\mathrm{ABCD}\end{array}$ & & -26 & 233 & -2.5 & -18 & 237 & -1.7 & & & & & & \\
\hline Estacio & 5 & \multicolumn{3}{|c|}{ enalapril } & & isold & & & & $\mathrm{CC}$ & AER & & $85^{1}$ \\
\hline $\begin{array}{l}2000[58] \\
\mathrm{ABCD}\end{array}$ & & -33 & 234 & -1.9 & 0 & 231 & -1.8 & & & & & & \\
\hline Gaede & 8 & & stand & & & inten & & & & ${ }^{51} \mathrm{Cr}-\mathrm{EDTA}$ & AER & 117 & \\
\hline $\begin{array}{l}1999[26] \\
2003[25]\end{array}$ & & 3.8 & 80 & -2.9 & -7.4 & 80 & -2.5 & & & & & & \\
\hline Nankervis & 3 & & erind & & & place & & & & CG & AER & & 94 \\
\hline 1998 [59] & & -16.8 & 20 & -2.9 & 16.4 & 20 & -2.1 & & & & & & \\
\hline
\end{tabular}

$\mathrm{PP}=$ Proteinuria parameter; $\mathrm{CC}=$ creatinine clearance; $\mathrm{n}=$ number of patients; $\Delta \mathrm{AER} \%=$ initial percentage change in AER or equivalent during first year of study; $\Delta$ GFR $\%$ = annual percentage change in GFR or equivalent assessed over entire study period.

${ }^{1}$ At baseline, the relative proportion of participants in the ABCD study (hypertensive arm) was normo 49\%, micro 32\% and macro 19\%, with an overall mean AER of $32 \mu \mathrm{g} / \mathrm{min}$. 
Table 2d. Type 1, late DN

\begin{tabular}{|c|c|c|c|c|c|c|c|c|c|c|c|c|c|c|}
\hline \multirow[t]{2}{*}{ Author } & \multirow{2}{*}{$\begin{array}{l}\text { Study } \\
\text { duration } \\
\text { years }\end{array}$} & \multicolumn{9}{|c|}{ Intervention } & \multirow{2}{*}{$\begin{array}{l}\text { GFR } \\
\text { method }\end{array}$} & \multirow[t]{2}{*}{ PP } & \multicolumn{2}{|c|}{ Mean baseline GFR } \\
\hline & & $\begin{array}{l}\Delta \text { AER } \\
\%\end{array}$ & $\mathrm{n}$ & $\begin{array}{l}\Delta \text { GFR } \\
\%\end{array}$ & $\begin{array}{l}\Delta \text { AER } \\
\%\end{array}$ & $\mathrm{n}$ & $\begin{array}{l}\Delta \text { GFR } \\
\%\end{array}$ & $\begin{array}{l}\Delta \text { AER } \\
\%\end{array}$ & $\mathrm{n}$ & $\begin{array}{l}\Delta \text { GFR } \\
\%\end{array}$ & & & $\begin{array}{l}\mathrm{ml} / \mathrm{min} / \\
1.73 \mathrm{~m}^{2}\end{array}$ & $\begin{array}{l}\mathrm{ml} / \\
\mathrm{min}\end{array}$ \\
\hline $\begin{array}{l}\text { Mogensen } \\
1982[18]\end{array}$ & 6.1 & 25.8 & $\begin{array}{c}\text { pre- } \\
5\end{array}$ & -16.6 & 1.6 & $\begin{array}{r}\operatorname{urin} \\
5\end{array}$ & $x^{1}-6.2$ & & & & $\begin{array}{l}{ }^{125} \mathrm{I}- \\
\text { iotha- } \\
\text { lamate }\end{array}$ & AER & & 86 \\
\hline $\begin{array}{l}\text { Parving } \\
1987 \text { [19] }\end{array}$ & $\begin{array}{l}2 \text { years (pre) } \\
6 \text { years (post) }\end{array}$ & 50.0 & $\begin{array}{l}\text { pre- } \\
10\end{array}$ & -11.2 & $\begin{array}{l}\text { met } \\
-38.0\end{array}$ & $\begin{array}{c}\mathrm{op} / \mathrm{fr}_{\mathrm{r}} \\
10\end{array}$ & $\begin{array}{l}\text { HCT } \\
-4.0\end{array}$ & & & & ${ }^{51} \mathrm{Cr}-\mathrm{EDTA}$ & AER & $\begin{array}{r}102 \text { (pre-Tx } \\
80 \text { (Tx gp) }\end{array}$ & \\
\hline $\begin{array}{l}\text { Parving } \\
1988 \text { [60] }\end{array}$ & 2.3 & 5.0 & $\begin{array}{l}\text { untre } \\
13\end{array}$ & $\begin{array}{l}\text { ted } \\
-10.4\end{array}$ & -39.0 & $\begin{array}{c}\text { apto } \\
18\end{array}$ & il -5.9 & & & & ${ }^{51} \mathrm{Cr}$-EDTA & AER & 97 & \\
\hline $\begin{array}{l}\text { Bjorck } \\
1992[61]\end{array}$ & 2.25 & $\begin{array}{l}\text { meto } \\
10.7\end{array}$ & $\begin{array}{l}\text { prolc } \\
18\end{array}$ & $\begin{array}{l} \pm \text { frus } \\
-11.7\end{array}$ & $\begin{array}{l}\text { en } \\
-68.8\end{array}$ & $\begin{array}{c}\text { april } \\
22\end{array}$ & $\begin{array}{l}\text { frus } \\
\quad-4.3\end{array}$ & & & & ${ }^{51} \mathrm{Cr}-\mathrm{EDTA}$ & AER & 47 & \\
\hline $\begin{array}{l}\text { Lewis } \\
1993[5]\end{array}$ & 2.7 & $2.0^{\text {plac }}$ & $\begin{array}{l}\text { cebo } \\
202\end{array}$ & $\begin{array}{c}\text { conv'l } \\
-17.0\end{array}$ & $\begin{array}{l}\text { cap } \\
-25.0\end{array}$ & $\begin{array}{l}\text { pril } \\
207\end{array}$ & $\begin{array}{l}\text { :onv'l } \\
-11.0\end{array}$ & & & & CC & $\begin{array}{l}\text { total } \\
\text { pro- } \\
\text { teinuria }\end{array}$ & & 82 \\
\hline
\end{tabular}

$\mathrm{PP}=$ Proteinuria parameter; $\mathrm{CC}=$ creatinine clearance; $\mathrm{n}=$ number of patients; $\Delta$ AER $\%=$ initial percentage change in AER or equivalent during first year of study; $\Delta$ GFR $\%=$ annual percentage change in GFR or equivalent assessed over entire study period; pre- $R_{x}=$ pretreatment; during $R_{x}=$ during treatment; conv'l = conventional.

${ }^{1}$ Propranolol or metoprolol, \pm hydralazine, prazosin or frusemide.

Table 2e. Type 2, late DN

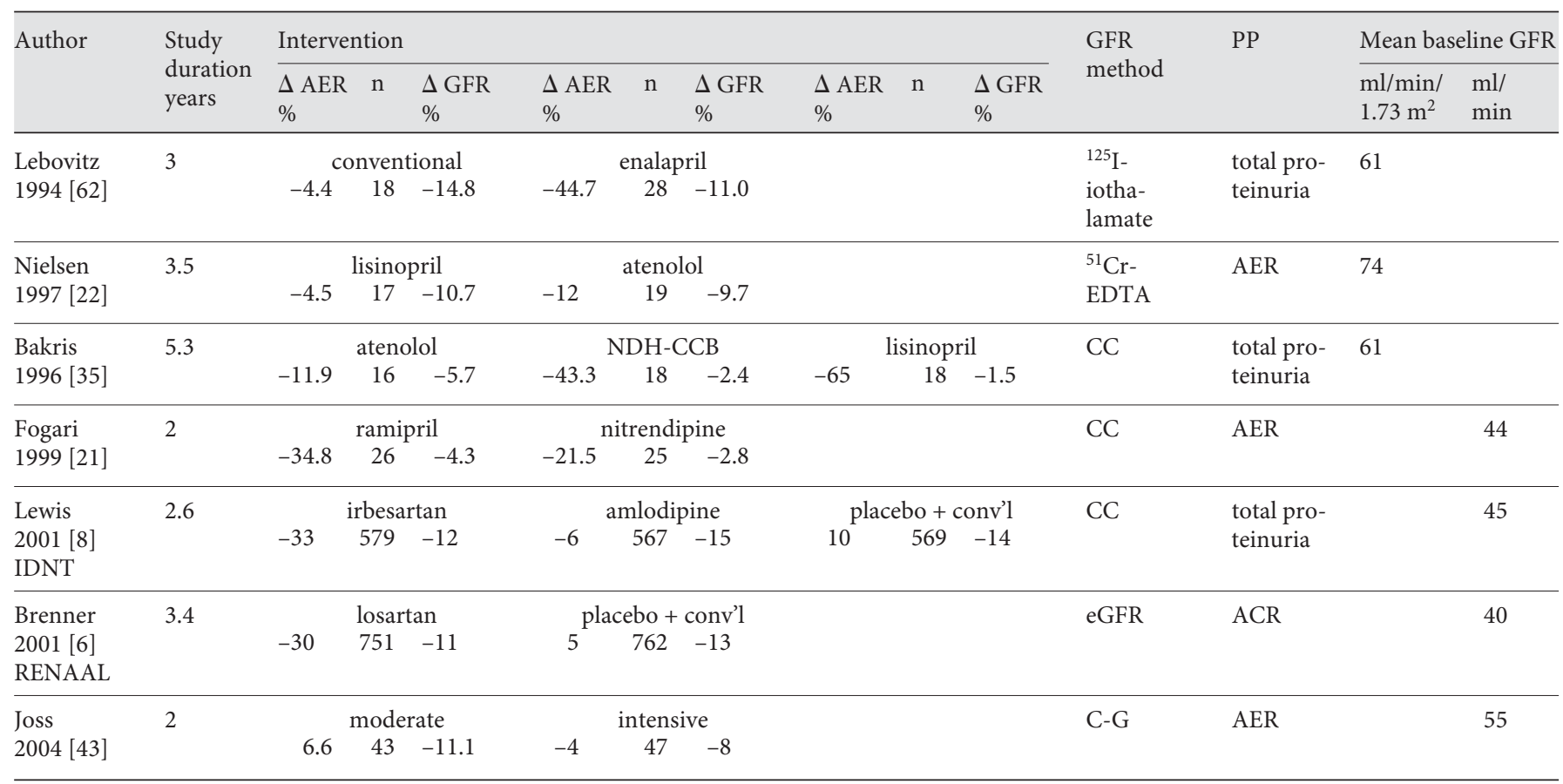

$\mathrm{PP}=$ Proteinuria parameter; $\mathrm{CC}=$ creatinine clearance; $\mathrm{n}=$ number of patients; $\Delta$ AER $\%=$ initial percentage change in AER or equivalent during first year of study; $\Delta$ GFR $\%$ = annual percentage change in GFR or equivalent assessed over entire study period. 
Fig. 1. Baseline renal parameters. a AER = Baseline albumin excretion rate or equivalent in individual study groups. $200 \mu \mathrm{g} /$ $\mathrm{min}=$ AER $300 \mathrm{mg} / 24 \mathrm{~h}=$ total protein uria $500 \mathrm{mg} / 24 \mathrm{~h}=300 \mathrm{mg}$ albumin per gram of creatinine. $\mathbf{b}$ GFR = Baseline glomerular filtration rate in individual study groups. $\bigcirc=$ GFR $\mathrm{ml} / \mathrm{min} / 1.73 \mathrm{~m}^{2}, \bullet=$ GFR $\mathrm{ml} / \mathrm{min}, \mathrm{NT}=$ normotensive, $\mathrm{HT}=$ hypertensive.

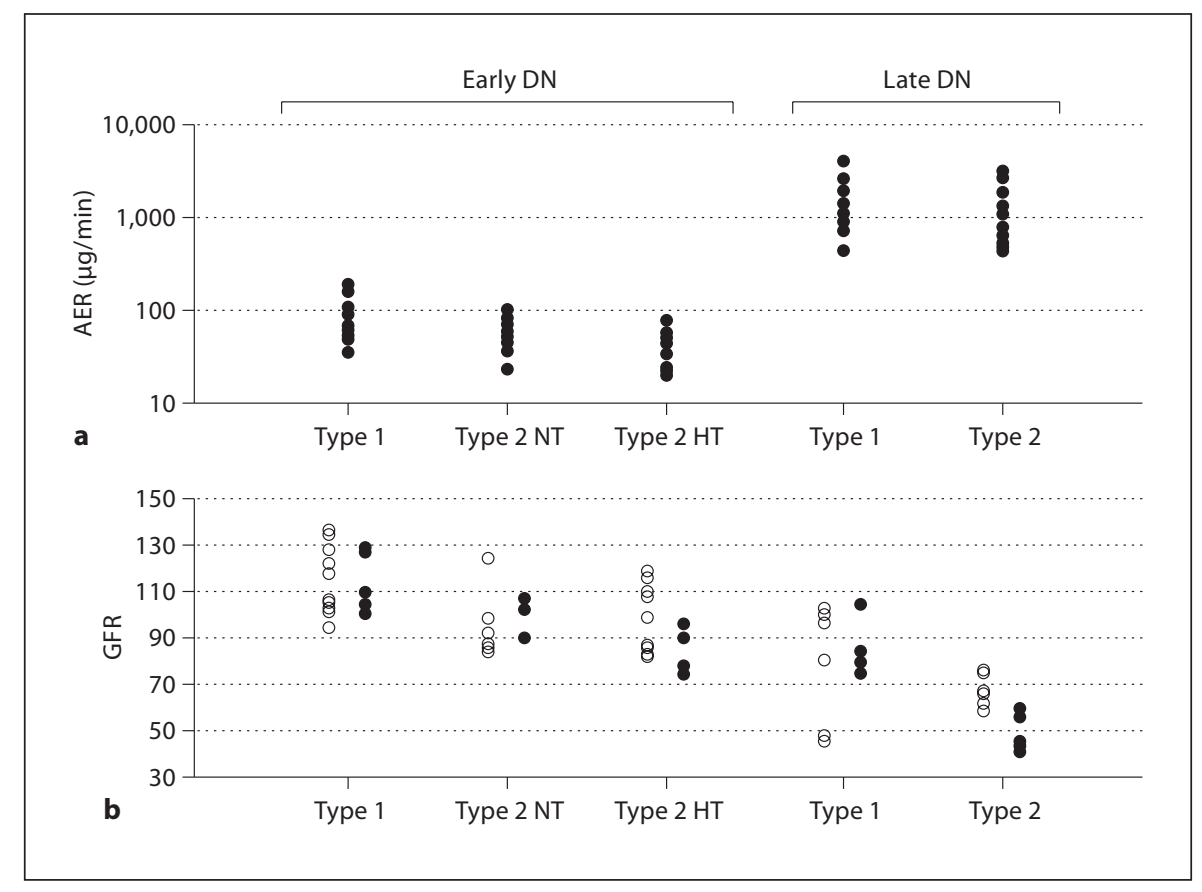

In five studies with 10 treatment groups of type 1 diabetes and late nephropathy, there was a close relationship between initial change in albuminuria during the first year and the mean rate of decline in GFR during the whole study in each treatment group $(\mathrm{r}=-0.67, \mathrm{p}=0.034)$ (fig. 3). The percentage changes in AER and GFR were also compared by a weighted comparison of means to allow for differences in sample size [20]. This showed that the weighted mean initial change in AER was $-12.7 \%$, and the weighted mean overall change in GFR was $-12.8 \%$. In seven studies with 16 treatment groups of patients with type 2 diabetes and late nephropathy, there was a significant relationship between initial change in albuminuria and the overall mean rate of decline in GFR for each study group $(\mathrm{r}=-0.57, \mathrm{p}=0.02)$ (fig. 4). The weighted mean initial change in AER was $-11.8 \%$ and the weighted mean overall change in GFR was $-12.5 \%$.

In contrast with results in late nephropathy, studies of antihypertensive therapy in early nephropathy did not show a significant relationship between initial changes in albuminuria and long-term changes renal function. As shown in figure 3, in nine studies with 23 treatment groups in normotensive patients with type 1 diabetes and early nephropathy, there was no significant relationship between initial changes in albuminuria and long-term change in GFR ( $r=-0.10, p=0.33)$. In five studies with 11 treatment groups in normotensive patients with type
2 diabetes and early nephropathy (fig. 4), there was also no significant relationship between initial changes in albuminuria and long-term rate of decline in GFR ( $r=$ $-0.18, p=0.30$ ). In seven studies with 17 treatment groups of hypertensive patients with type 2 diabetes and early nephropathy (fig. 4), initial changes in albuminuria were not significantly related to long-term change in GFR ( $\mathrm{r}=$ $+0.02, \mathrm{p}=0.47$ ).

In order to further clarify the contribution of RAS inhibitor treatment groups and their corresponding control or comparator groups, studies of late nephropathy in type 1 diabetes and late nephropathy in type 2 diabetes have been replotted to show the relationship of each RAS inhibitor study with the corresponding control or comparator group (fig. 5).

Because of limitations due to small numbers of study groups, it is difficult to separate RAS inhibitor studies from the rest. However, the overall trend of the data suggests that the relationship between $\Delta$ AER and $\Delta$ GFR is not necessarily class-specific. For instance, two small studies performed in the 1980s by Mogensen [18] and Parving et al. [19] showed marked attenuation of GFR decline after commencement of non-RAS inhibitor therapy.

The relationship between mean arterial pressure, expressed as final attained pressure or $\%$ decline during the study, and rate of annual decline of GFR was also assessed. No significant relationship was observed between 
Fig. 2. Initial changes in AER and annual changes in GFR according to diabetes type and stage of nephropathy. a $\% \Delta$ AER $=$ Percentage change in albuminuria or equivalent in individual study groups during first year of study (mean \pm SEM). b $\% \Delta \mathrm{GFR}=$ Annual percentage change in GFR or equivalent in individual study groups, assessed over the entire study pe$\operatorname{riod}($ mean $\pm S E M)$.

Fig. 3. Relationship between initial change in AER and overall change in GFR per year in type 1 diabetes. a Mean data from 23 study groups in 9 studies of type 1 diabetes and early diabetic nephropathy are shown. b Mean data from 10 study groups in 5 studies of type 1 diabetes and late diabetic nephropathy are shown.

Renal Response to Antihypertensive Therapy in Diabetes
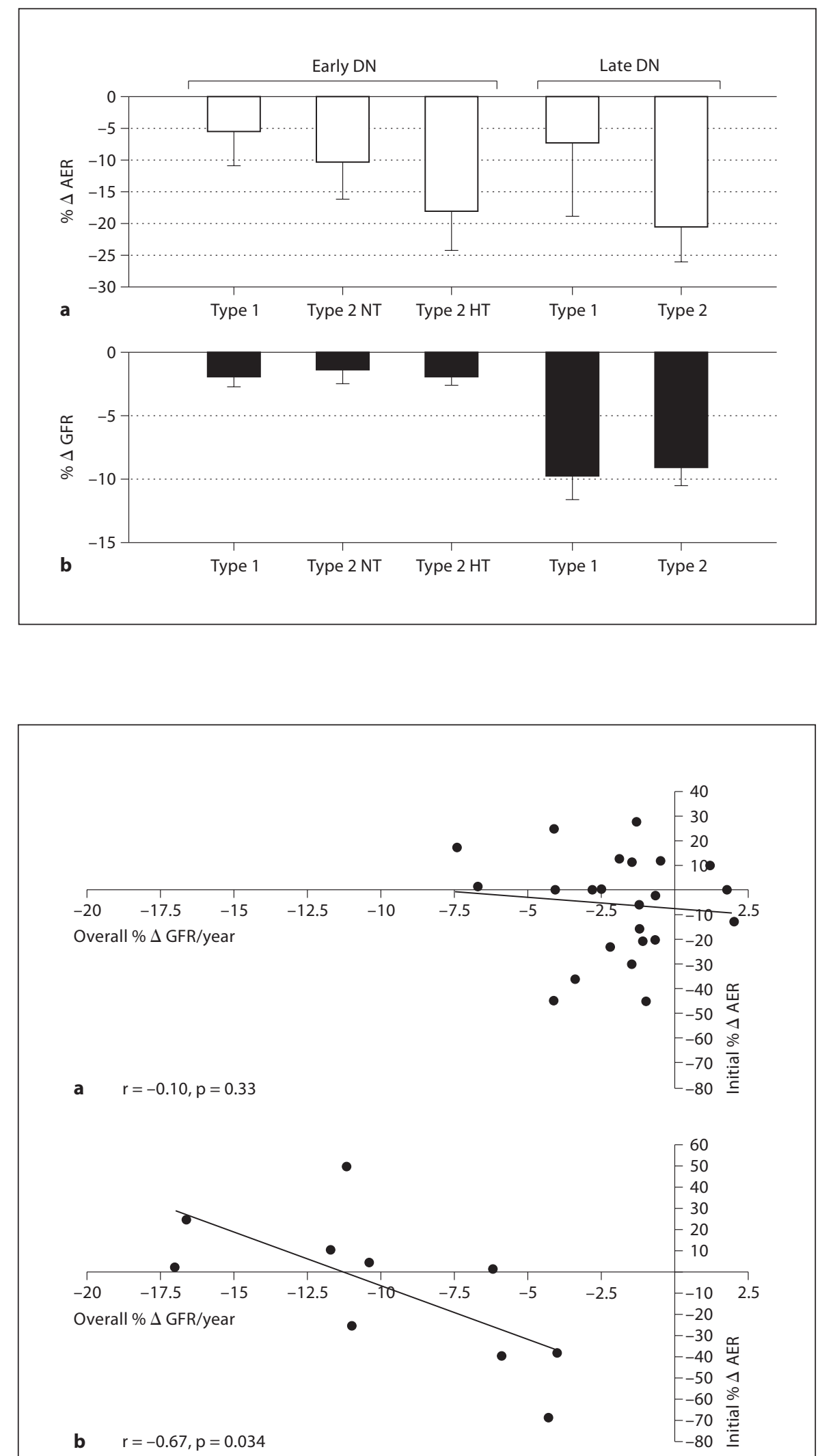
Fig. 4. Relationship between initial change in AER and overall change in GFR per year in type 2 diabetes. a Mean data from 11 study groups in 5 studies of type 2 diabetes and normotensive early diabetic nephropathy are shown. b Mean data from 17 study groups in 7 studies of type 2 diabetes and hypertensive early diabetic nephropathy are shown. c Mean data from 16 study groups in 7 studies of type 2 diabetes and late diabetic nephropathy are shown.
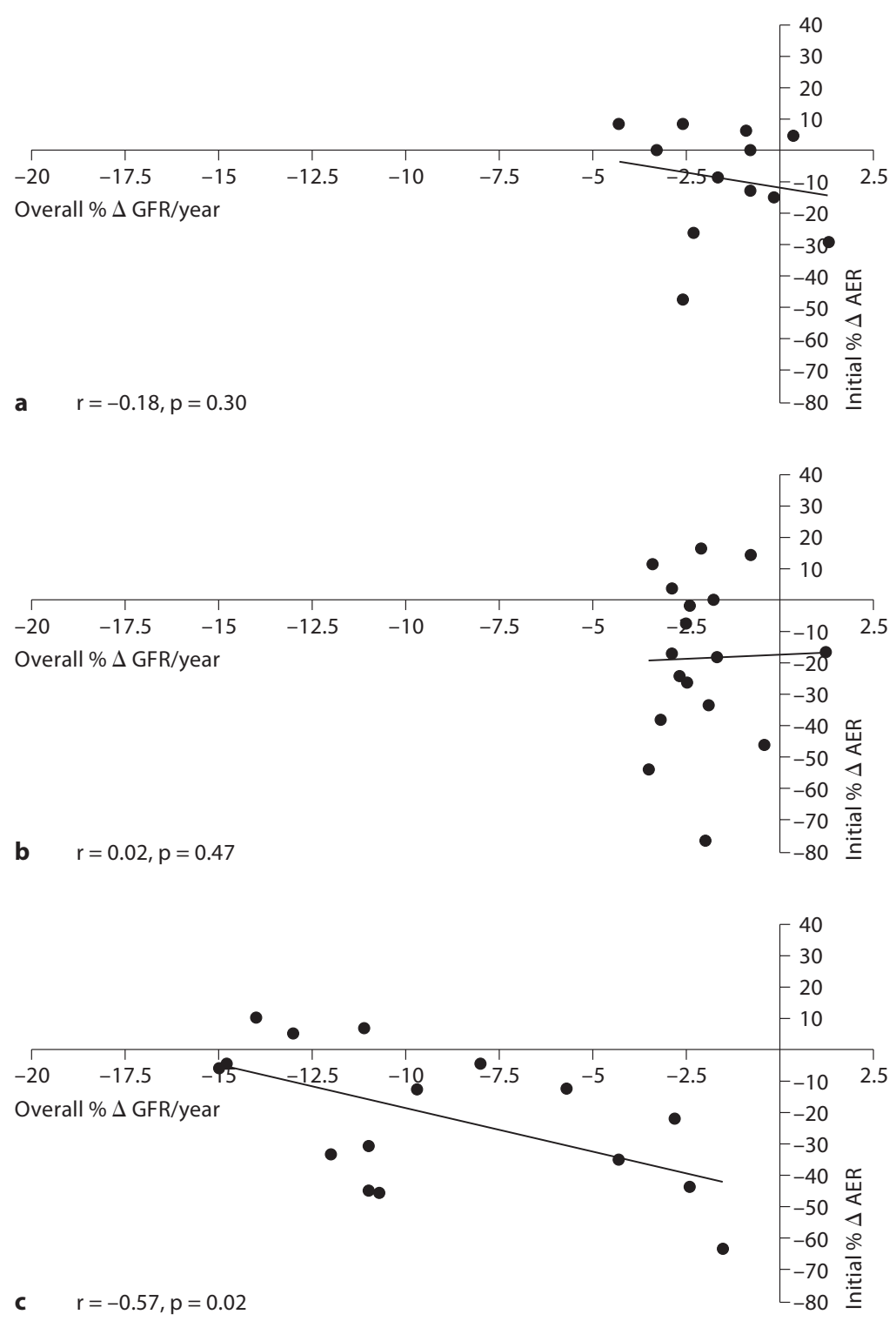

Table 3. Absolute rates of decline of GFR according to study category

\begin{tabular}{llllll}
\hline & $\begin{array}{l}\text { Type } 1 \\
\text { early }\end{array}$ & $\begin{array}{l}\text { Type 2 } \\
\text { early NT }\end{array}$ & $\begin{array}{l}\text { Type 2 } \\
\text { early HT }\end{array}$ & $\begin{array}{l}\text { Type 1 } \\
\text { late }\end{array}$ & $\begin{array}{l}\text { Type 2 } \\
\text { late }\end{array}$ \\
\hline$\Delta$ GFR, ml/min/year & $\begin{array}{l}0.94 \pm 0.87 \\
(\mathrm{n}=5)\end{array}$ & $\begin{array}{l}0.63 \pm 0.79^{* *} \\
(\mathrm{n}=4)\end{array}$ & $\begin{array}{l}1.58 \pm 0.61^{* *} \\
(\mathrm{n}=6)\end{array}$ & $\begin{array}{l}10.35 \pm 1.57 \\
(\mathrm{n}=4)\end{array}$ & $\begin{array}{l}4.68 \pm 0.65 \\
(\mathrm{n}=9)\end{array}$ \\
\hline$\Delta$ GFR, ml/min/1.73 $\mathrm{m}^{2} /$ year & $\begin{array}{l}2.42 \pm 0.57^{* *} \\
(\mathrm{n}=18)\end{array}$ & $\begin{array}{l}2.09 \pm 0.44^{*} \\
(\mathrm{n}=7)\end{array}$ & $\begin{array}{l}2.21 \pm 0.29^{* *} \\
(\mathrm{n}=11)\end{array}$ & $\begin{array}{l}6.26 \pm 1.47 \\
(\mathrm{n}=6)\end{array}$ & $\begin{array}{l}5.32 \pm 1.27 \\
(\mathrm{n}=7)\end{array}$ \\
\hline
\end{tabular}

$\mathrm{n}=$ Number of study groups.

${ }^{*} \mathrm{p}<0.05$ vs. corresponding late; ${ }^{* *} \mathrm{p}<0.01$ vs. corresponding late. 
Fig. 5. Relationship between change in AER and overall change in GFR per year in studies of late nephropathy according to type of antihypertensive therapy in type 1 diabetes (a) and type 2 diabetes (b). Treatment groups for each study are linked by a solid line.
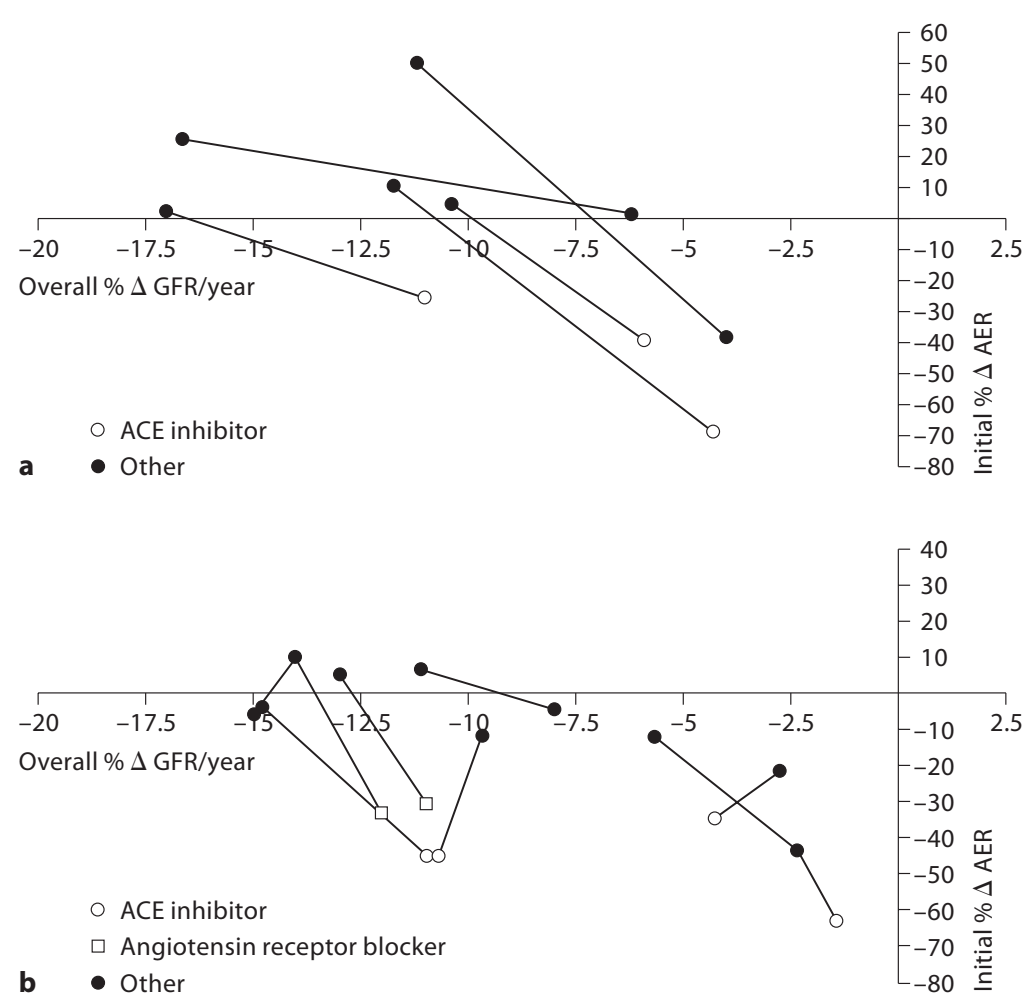

any of these parameters and rate of decline of GFR in early nephropathy. In a small number of study groups of late nephropathy in type 1 diabetes, there was a correlation between final mean arterial pressure and annual change in GFR expressed as $\mathrm{ml} / \mathrm{min} / 1.73 \mathrm{~m}^{2}(\mathrm{r}=-0.92$, $\mathrm{p}$ $<0.01, n=6$ ). The corresponding correlation was not significant in studies of late nephropathy in type 2 diabetes. In 14 study groups of late nephropathy in type 2 diabetes, the change in mean arterial pressure during the study was correlated with the percentage change in GFR $(r=$ $-0.56, \mathrm{p}<0.05)$, but this correlation was not significant in studies of late nephropathy in type 1 diabetes.

\section{Discussion}

In studies of late nephropathy in both type 1 and type 2 diabetes, this analysis demonstrates a strong association between initial changes in albuminuria and overall changes in GFR during antihypertensive therapy. This is consistent with the concept that proteinuria itself is a major progression promoter in established diabetic nephropathy [23].
In contrast with the results in late nephropathy, there was no significant relationship between the initial AER and overall GFR responses in studies of early diabetic nephropathy. This raises the possibility that treatmentrelated decreases in albuminuria in early nephropathy, with normal or near-normal GFR, represent functional and presumably reversible changes in renal albumin handling and/or GFR, which may be independently modulated.

Because of disparities in study size, it was important to determine if data obtained in larger studies were representative of the overall results obtained. The captopril study by the Collaborative Study Group [5] assessed the effects of captopril in over 400 patients with type 1 diabetes and late nephropathy and was consistent with the general trend of results in other studies in this category (fig. 3). Results from over 1,500 patients with type 2 diabetes in the IDNT study [8] and over 1,400 patients with type 2 diabetes in the RENAAL study [6] were also consistent with the overall results shown in studies of type 2 diabetes and late nephropathy (fig. 4). AER and GFR responses, expressed as weighted means, were almost identical in studies of late nephropathy in type 1 and type 2

Am J Nephrol 2008;28:614-627 
diabetes. This implies similar pathogenetic relationships between AER and GFR during overt nephropathy in both types of diabetes.

In early diabetic nephropathy, results from two large studies reflected the overall results. The microalbuminuria captopril study in normotensive patients with type 1 diabetes and microalbuminuria with over 100 participants, showed that an initial decrease in albuminuria of $30 \%$, similar to that documented in percentage terms in patients with overt nephropathy, was not associated with a significant decrease in GFR over 2 years when assessed by creatinine clearance [10]. A sub-study using isotopic determination of GFR showed similar results [24]. In the IRMA 2 study of hypertensive patients with type 2 diabetes and microalbuminuria, an initial decrease of $38 \%$ in AER in patients treated with irbesartan $300 \mathrm{mg}$ per day was not associated with a significant change in creatinine clearance over two years [11].

The longest study of intensive therapy in hypertensive patients with type 2 diabetes and microalbuminuria was performed over 8 years in 160 patients in the STENO-2 study [25]. Intensive multifactorial intervention focusing on antihypertensive therapy, glycemic control and control of dyslipidemia, was shown to nearly halve the rate of progression to macroalbuminuria when compared with results of standard therapy [26]. However, despite a follow-up of 8 years, the rate of decline of GFR of around $3 \mathrm{ml} / \mathrm{min} /$ year was similar in the intensively treated group and the group receiving standard therapy [25]. Analysis of the antialbuminuric response and the longterm GFR response within each study group by intention-to-treat analysis was representative of the general trend of data for hypertensive patients with microalbuminuria and type 2 diabetes. By contrast, a post-hoc analysis of pooled data for the intensive and standard groups showed that patients progressing from micro- to macroalbuminuria had a greater rate of decline of GFR over 8 years compared with patients who remained microalbuminuric or who regressed to normoalbuminuria [27]. The results of the STENO-2 study indicate that early decreases in AER during multifactorial intervention in hypertensive patients with microalbuminuria and normal or near-normal baseline GFR are not necessarily reflected in a slower rate of decline in GFR when examined prospectively on an intention-to-treat basis over 8 years [25]. This is consistent with the results of other studies in early nephropathy.

The failure to demonstrate a link between changes in albuminuria and renal function during antihypertensive therapy in early nephropathy studies may be due to limi- tations in study design or to biological factors. In support of study design as an explanation is the slow rate of decline of GFR in early nephropathy, which may lead to errors in estimation or measurement of changes in GFR. However, the failure to show prospectively that changes in AER in the STENO-2 study [25] predict long-term decline in GFR in hypertensive microalbuminuric patients over 8 years argues against this. In this study, there were equal numbers of cardiovascular deaths over 8 years $(7 / 80)$ in the groups receiving conventional or intensive therapy. Nevertheless, studies longer than this may be confounded by survival bias due to cardiovascular deaths.

A biological explanation for the lack of correlation between albuminuria response and rate of decline in GFR could be provided by differences in renal protein handling mechanisms in early compared with late nephropathy, even though percentage changes in AER were similar in hypertensive patients with type 2 diabetes with early and late nephropathy. In early nephropathy, initial changes in AER induced by antihypertensive agents are likely to reflect predominantly functional changes in glomerular and renal tubular handling of albumin by nephrons that are near-normal structurally [28]. The increase in AER shortly after cessation of renin angiotensin inhibition in studies of microalbuminuric patients supports this explanation [29-33]. Further evidence in support of independent regulation of AER and GFR in early nephropathy in patients with type 2 diabetes has been provided by the United Kingdom Prospective Diabetes Study [34]. In that study, early increases in AER and decreases in GFR occurred simultaneously in 14\% and independently in around $40 \%$ of patients when evaluated over 15 years from diagnosis.

The present analysis suggests that uncoupling of AER and GFR also occurs in an interventional context in patients with type 2 diabetes and early nephropathy. Previous studies with verapamil in hypertensive patients with type 2 diabetes lend support to the concept that the nature of the AER response to antihypertensive therapy differs before and after the onset of overt nephropathy. For instance, in patients with overt nephropathy, verapamil decreased proteinuria and ameliorated the rate of decline of renal function [35]. By contrast, in normoalbuminuric patients, verapamil had no effect in preventing the onset of microalbuminuria in a placebo-controlled study over 4 years [36].

In later stages of nephropathy, changes in AER and GFR are less reversible and are associated with renal ultrastructural damage. Antihypertensive therapy in late nephropathy induces early hemodynamic and later ul- 
trastructural effects. Early changes include lowering of intraglomerular pressure [37] and modulation of the glomerular filtration barrier by nephrin and other components of the slit pore diaphragm [38]. Renal ultrastructural changes could reflect a direct pathogenetic link between AER and GFR, but it is also possible that changes in AER and GFR are concurrent and not causally related. A direct pathogenetic role for renal tubular uptake of albumin has been proposed as a mediator of renal damage and eventual loss of renal function [23]. Reduction in AER by antihypertensive therapy would therefore be expected to reduce the rate of decline of GFR. A further consideration is that changes in AER reflect not only glomerular filtration of albumin but also renal tubular processing of albumin [39] whereas changes in GFR reflect alterations in renal hemodynamic and/or glomerular ultrastructure [28]. These diverse effects may not be directly linked to each other in a causal sense. However, there is experimental and clinical evidence to show that AER and GFR are both modulated by angiotensin [40].

It is important to note that changes in blood pressure occurred concurrently with changes in AER and GFR in all studies. It follows that initial changes in AER reflect a combination of systemic and intrarenal effects of antihypertensive therapy. Both effects are likely to contribute to the long-term rate of decline of GFR in studies of late diabetic nephropathy.

This study has several potential methodological limitations. Firstly, it is based on a post-hoc analysis of group mean data in studies ranging over 30 years from the earlier studies in the late 1970s. Ideally, individual subject data from all eligible studies should have been included in a formal meta-analysis. This was not possible because of methodological differences in assessing proteinuria and in measuring or estimating GFR.

A further problem is the slow rate of GFR decline in early diabetic nephropathy. Most studies of early nephropathy were performed with follow-up of 2-4 years. In some of these, the rate of decline of GFR was close to the age expected decline in GFR in older patients. The design of future studies should recognize not only duration of follow-up but also evidence of progressive decline in renal function.

In order to achieve greater understanding of the relationship between albuminuria and GFR during antihypertensive therapy, further long-term studies are required in patients with GFR in the range of $90-45 \mathrm{ml} /$ min (CKD stage 2 and early 3 ). This indicates CKD that is more advanced than in most previous studies of microalbuminuria but less advanced than in previous studies of established nephropathy. Accurate measurement of rates of decline of GFR in such patients would help to define the full spectrum of renal responses to RAS inhibitors and other antihypertensive agents. This is less easily achieved in endpoint studies, which focus on fast trackers by assessing rates of conversion from micro- to macroalbuminuria or rates of doubling of serum creatinine [41].

In summary, this analysis of 12 studies ( 26 treatment groups) of antihypertensive therapy in late diabetic nephropathy confirms that the initial decrease in AER can be used as a predictor or surrogate for the subsequent rate of decline of GFR. By contrast, this relationship could not be demonstrated on an intention-to-treat basis in 21 studies (51 treatment groups) of early nephropathy with a follow-up of 2-8 years. This implies a change in the relationship between AER and GFR as nephropathy progresses from early to late stages. It follows that assessment of renoprotection during antihypertensive therapy of early diabetic nephropathy should be based not only on albuminuria but should also include evaluation of the GFR response.

\section{Acknowledgement}

This study was supported by NHMRC grant No 266505 'Albuminuric and nonalbuminuric pathways to renal impairment in diabetic nephropathy'.

\section{References}

1 Bakris GL, Williams M, Dworkin L, Elliott WJ, Epstein M, Toto R, Tuttle K, Douglas J, Hsueh W, Sowers J: Preserving renal function in adults with hypertension and diabetes: a consensus approach. National Kidney Foundation Hypertension and Diabetes Executive Committees Working Group. Am J Kidney Dis 2000;36:646-661.
Rossing P, Hommel E, Smidt UM, Parving $\mathrm{HH}$ : Reduction in albuminuria predicts a beneficial effect on diminishing the progression of human diabetic nephropathy during antihypertensive treatment. Diabetologia 1994;37:511-516.
3 Apperloo AJ, de Zeeuw D, de Jong PE: Shortterm antiproteinuric response to antihypertensive treatment predicts long-term GFR decline in patients with non-diabetic renal disease. Kidney Int Suppl 1994;45:S174S178. 
-4 Gansevoort RT, de Zeeuw D, Shahinfar S, Redfield A, de Jong PE: Effects of the angiotensin II antagonist losartan in hypertensive patients with renal disease. J Hypertens Suppl 1994;12:S37-S42.

$\checkmark 5$ Lewis EJ, Hunsicker LG, Bain RP, Rohde RD: The effect of angiotensin-converting-enzyme inhibition on diabetic nephropathy. The Collaborative Study Group. N Engl J Med 1993;329:1456-1462.

-6 Brenner BM, Cooper ME, de Zeeuw D, Keane WF, Mitch WE, Parving $\mathrm{HH}, \mathrm{Re}$ muzzi G, Snapinn SM, Zhang Z, Shahinfar S: Effects of losartan on renal and cardiovascular outcomes in patients with type 2 diabetes and nephropathy. N Engl J Med 2001;345: 861-869.

$\checkmark 7$ De Zeeuw D, Remuzzi G, Parving $\mathrm{HH}$, Keane WF, Zhang Z, Shahinfar S, Snapinn S, Cooper ME, Mitch WE, Brenner BM: Proteinuria, a target for renoprotection in patients with type 2 diabetic nephropathy: lessons from RENAAL. Kidney Int 2004;65: 2309-2320.

-8 Lewis EJ, Hunsicker LG, Clarke WR, Berl T, Pohl MA, Lewis JB, Ritz E, Atkins RC, Rohde $\mathrm{R}, \mathrm{Raz}$ I: Renoprotective effect of the angiotensin-receptor antagonist irbesartan in patients with nephropathy due to type 2 diabetes. N Engl J Med 2001;345:851-860.

$\checkmark$ Atkins RC, Briganti EM, Lewis JB, Hunsicker LG, Braden G, Champion de Crespigny PJ, DeFerrari G, Drury P, Locatelli F, Wiegmann TB, Lewis EJ: Proteinuria reduction and progression to renal failure in patients with type 2 diabetes mellitus and overt nephropathy. Am J Kidney Dis 2005;45:281287.

10 The Microalbuminuria Captopril Study Group: Captopril reduces the risk of nephropathy in IDDM patients with microalbuminuria. Diabetologia 1996;39:587-593.

- 11 Parving $\mathrm{HH}$, Lehnert H, Brochner-Mortensen J, Gomis R, Andersen S, Arner P: The effect of irbesartan on the development of diabetic nephropathy in patients with type 2 diabetes. N Engl J Med 2001;345:870-878.

-12 Strippoli GF, Craig M, Deeks JJ, Schena FP, Craig JC: Effects of angiotensin converting enzyme inhibitors and angiotensin II receptor antagonists on mortality and renal outcomes in diabetic nephropathy: systematic review. BMJ 2004;329:828-831.

13 Viberti GC, Walker JD, Pinto J: Diabetic Nephropathy; in Alberti K, DeFronzo R, Keen $\mathrm{H}$, Zimmet P (eds): International Textbook of Diabetes Mellitus. Chichester, Wiley, 1992, vol 2, pp 1267-1328.

-14 Parving HH, Hommel E, Jensen BR, Hansen HP: Long-term beneficial effect of ACE inhibition on diabetic nephropathy in normotensive type 1 diabetic patients. Kidney Int 2001;60:228-234.
15 Premaratne E, MacIsaac RJ, Tsalamandris C, Panagiotopoulos S, Smith T, Jerums G: Renal hyperfiltration in type 2 diabetes: effect of age-related decline in glomerular filtration rate. Diabetologia 2005;48:2486-2493.

16 Velussi M, Brocco E, Frogato F, Zolli M, Muollo B, Maioli M, Carraro A, Tonolo G, Fresu P, Cernigoi AM, Fioretto P, Nosadini $\mathrm{R}$ : Effects of cilazapril and amlodipine on kidney function in hypertensive NIDDM patients. Diabetes 1996;45:216-222.

17 Cordonnier DJ, Pinel N, Barro C, Maynard M, Zaoui P, Halimi S, de Ligny BH, Reznic Y, Simon D, Bilous RW: Expansion of cortical interstitium is limited by converting enzyme inhibition in type 2 diabetic patients with glomerulosclerosis. The Diabiopsies Group. J Am Soc Nephrol 1999;10:1253-1263.

18 Mogensen CE: Long-term antihypertensive treatment inhibiting progression of diabetic nephropathy. Br Med J (Clin Res Ed) 1982; 285:685-688.

19 Parving HH, Andersen AR, Smidt UM, Hommel E, Mathiesen ER, Svendsen PA: Effect of antihypertensive treatment on kidney function in diabetic nephropathy. Br Med J (Clin Res Ed) 1987;294:1443-1447.

20 Bland JM, Kerry SM: Statistics notes. Weighted comparison of means. BMJ 1998;316:129.

-21 Fogari R, Zoppi A, Corradi L, Mugellini A, Lazzari P, Preti P, Lusardi P: Long-term effects of ramipril and nitrendipine on albuminuria in hypertensive patients with type II diabetes and impaired renal function. J Hum Hypertens 1999; 13:47-53.

22 Nielsen FS, Rossing P, Gall MA, Skott P, Smidt UM, Parving HH: Long-term effect of lisinopril and atenolol on kidney function in hypertensive NIDDM subjects with diabetic nephropathy. Diabetes 1997;46:1182-1188.

23 Remuzzi G, Bertani T: Pathophysiology of progressive nephropathies. N Engl J Med 1998;339:1448-1456.

24 Viberti G, Mogensen CE, Groop LC, Pauls JF: Effect of captopril on progression to clinical proteinuria in patients with insulindependent diabetes mellitus and microalbuminuria. European Microalbuminuria Captopril Study Group. JAMA 1994;271: 275-279.

25 Gaede P, Vedel P, Larsen N, Jensen GV, Parving $\mathrm{HH}$, Pedersen $\mathrm{O}$ : Multifactorial intervention and cardiovascular disease in patients with type 2 diabetes. N Engl J Med 2003;348:383-393.

26 Gaede P, Vedel P, Parving HH, Pedersen O: Intensified multifactorial intervention in patients with type 2 diabetes mellitus and microalbuminuria: the STENO type 2 randomised study. Lancet 1999;353:617-622.

27 Gaede P, Tarnow L, Vedel P, Parving HH, Pedersen O: Remission to normoalbuminuria during multifactorial treatment preserves kidney function in patients with type 2 diabetes and microalbuminuria. Nephrol Dial Transplant 2004;19:2784-2788.
28 Mauer SM, Steffes MW, Ellis EN, Sutherland DE, Brown DM, Goetz FC: Structural-functional relationships in diabetic nephropathy. J Clin Invest 1984;74:1143-1155.

29 Hansen HP, Nielsen FS, Rossing P, Jacobsen $\mathrm{P}$, Jensen BR, Parving $\mathrm{HH}$ : Kidney function after withdrawal of long-term antihypertensive treatment in diabetic nephropathy. Kidney Int Suppl 1997;63:S49-S53.

30 Anderson S, Brochner-Mortensen J, Parving $\mathrm{HH}$ : Kidney function during and after withdrawal of long-term irbesartan treatment in patients with type 2 diabetes and microalbuminuria. Diabetes Care 2003;26:3296-3302.

31 Melbourne Diabetic Nephropathy Study Group: Comparison between perindopril and nifedipine in hypertensive and normotensive diabetic patients with microalbuminuria. BMJ 1991;302:210-216.

32 Jerums G, Allen TJ, Campbell DJ, Cooper ME, Gilbert RE, Hammond JJ, O’Brien RC, Raffaele J, Tsalamandris C: Long-term renoprotection by perindopril or nifedipine in non-hypertensive patients with type 2 diabetes and microalbuminuria. Diabet Med 2004;21:1192-1199.

33 Passa P, Leblanc H, Chevrel A, Menard J: Discontinuing ACE inhibition in patients with diabetic nephropathy. BMJ 1991;302: 658.

34 Retnakaran R, Cull CA, Thorne KI, Adler AI, Holman RR: Risk factors for renal dysfunction in type 2 diabetes: UK Prospective Diabetes Study 74. Diabetes 2006;55:18321839.

35 Bakris GL, Copley JB, Vicknair N, Sadler R, Leurgans S: Calcium channel blockers versus other antihypertensive therapies on progression of NIDDM-associated nephropathy. Kidney Int 1996;50:1641-1650.

36 Ruggenenti P, Fassi A, Ilieva AP, Bruno S, Iliev IP, Brusegan V, Rubis N, Gherardi G, Arnoldi F, Ganeva M, Ene-Iordache B, Gaspari F, Perna A, Bossi A, Trevisan R, Dodesini AR, Remuzzi G: Preventing macroalbuminuria in type 2 diabetes. N Engl J Med 2004;351:1941-1951.

37 Zatz R, Dunn BR, Meyer TW, Anderson S, Rennke HG, Brenner BM: Prevention of diabetic glomerulopathy by pharmacological amelioration of glomerular capillary hypertension. J Clin Invest 1986;77:1925-1930.

38 Kriz W, Gretz N, Lemley KV. Progression of glomerular diseases: is the podocyte the culprit? Kidney Int 1998;54:687-697.

39 Russo LM, Sandoval RM, McKee M, Osicka TM, Collins AB, Brown D, Molitoris BA, Comper WD: The normal kidney filters nephrotic levels of albumin retrieved by proximal tubule cells: retrieval is disrupted in nephrotic states. Kidney Int 2007;71:504-513.

40 Cooper ME: Interaction of metabolic and haemodynamic factors in mediating experimental diabetic nephropathy. Diabetologia 2001;44:1957-1972. 
-41 Stevens L, Greene T, Levey A: Surrogate endpoints for clinical trials of kidney disease progression. Clin J Am Soc Nephrol 2006; 1: 874-884.

-42 Schrier RW, Estacio RO, Esler A, Mehler P. Effects of aggressive blood pressure control in normotensive type 2 diabetic patients on albuminuria, retinopathy and strokes. Kidney Int 2002;61:1086-1097.

-43 Joss N, Ferguson C, Brown C, Deighan CJ, Paterson KR, Boulton-Jones JM: Intensified treatment of patients with type 2 diabetes mellitus and overt nephropathy. Q J Med 2004;97:219-227.

44 Mathiesen ER, Hommel E, Giese J, Parving $\mathrm{HH}$ : Efficacy of captopril in postponing nephropathy in normotensive insulin-dependent diabetic patients with microalbuminuria. BMJ 1991;303:81-87.

45 Mathiesen ER, Hommel E, Hansen HP, Smidt UM, Parving HH: Randomised controlled trial of long-term efficacy of captopril on preservation of kidney function in normotensive patients with insulin-dependent diabetes and microalbuminuria. BMJ 1999; 319:24-25.

-46 Hansen KW, Klein F, Christensen PD, Sorensen K, Andersen PH, Moller J, Pedersen EB, Christiansen JS, Mogensen CE: Effects of captopril on ambulatory blood pressure, renal and cardiac function in microalbuminuric type 1 diabetic patients. Diabetes Metab 1994;20:485-493.

-47 Crepaldi G, Carta Q, Deferrari G, Mangili R, Navalesi R, Santeusanio F, Spalluto A, Vanasia A, Villa GM, Nosadini R: Effects of lisinopril and nifedipine on the progression to overt albuminuria in IDDM patients with incipient nephropathy and normal blood pressure. The Italian Microalbuminuria Study Group in IDDM. Diabetes Care 1998; 21:104-110.
48 O’Hare P, Bilbous R, Mitchell T, CJ OC, Viberti GC: Low-dose ramipril reduces microalbuminuria in type 1 diabetic patients without hypertension: results of a randomized controlled trial. Diabetes Care 2000;23: 1823-1829.

49 The European Study for the Prevention of Renal Disease in Type 1 Diabetes (ESPRIT): Effect of 3 years of antihypertensive therapy on renal structure in type 1 diabetic patients with albuminuria. Diabetes 2001;50:843850.

50 Jerums G, Allen TJ, Campbell DJ, Cooper ME, Gilbert RE, Hammond JJ, Raffaele J, Tsalamandris C: Long-term comparison between perindopril and nifedipine in normotensive patients with type 1 diabetes and $\mathrm{mi-}$ croalbuminuria. Am J Kidney Dis 2001;37: 890-899.

51 Bojestig M, Karlberg BE, Lindström T, Nystrom FH: Reduction of ACE activity is insufficient to decrease microalbuminuria in normotensive patients with type 1 diabetes. Diabetes Care 2001;24:919-924.

52 Poulsen PL, Ebbehoj E, Mogensen CE: Lisinopril reduces albuminuria during exercise in low grade microalbuminuric type 1 diabetic patients: a double-blind randomized study. J Intern Med 2001;249:433-440.

53 Sano T, Kawamura T, Matsumae H, Sasaki H, Nakayama M, Hara T, Matsuo S, Hotta N, Sakamoto N: Effects of long-term enalapril treatment on persistent microalbuminuria in well-controlled hypertensive and normotensive NIDDM patients. Diabetes Care. 1994;17:420-424.

54 Ahmad J, Siddiqui MA, Ahmad H: Effective postponement of diabetic nephropathy with enalapril in normotensive type 2 diabetic patients with microalbuminuria. Diabetes Care 1997;20:1576-1581.
5 Ravid M, Brosh D, Levi Z, Bar-Dayan Y, Ravid D, Rachmani R: Use of enalapril to attenuate decline in renal function in normotensive, normoalbuminuric patients with type 2 diabetes mellitus. A randomized, controlled trial. Ann Intern Med. 1998;128:982-988.

56 Lacourciere Y, Nadeau A, Poirier L, Tancrede G: Captopril or conventional therapy in hypertensive type II diabetics. Three-year analysis. Hypertension 1993;21:786-794.

57 Chan JC, Ko GT, Leung DH, Cheung RC, Cheung MY, So WY, Swaminathan R, Nicholls MG, Critchley JA, Cockram CS: Longterm effects of angiotensin-converting enzyme inhibition and metabolic control in hypertensive type 2 diabetic patients. Kidney Int 2000;57:590-600.

58 Estacio RO, Jeffers BW, Gifford N, Schrier RW: Effect of blood pressure control on diabetic microvascular complications in patients with hypertension and type 2 diabetes. Diabetes Care 2000;23(suppl 2):B54-B64.

59 Nankervis A, Nicholls K, Kilmartin G, Allen P, Ratnaike S, Martin FI: Effects of perindopril on renal histomorphometry in diabetic subjects with microalbuminuria: a 3-year placebo-controlled biopsy study. Metabolism 1998;47:12-15.

-60 Parving HH, Hommel E, Smidt UM: Protection of kidney function and decrease in albuminuria by captopril in insulin-dependent diabetics with nephropathy. BMJ 1988;297: 1086-1091.

61 Bjorck S, Mulec H, Johnsen SA, Norden G, Aurell M: Renal protective effect of enalapril in diabetic nephropathy. BMJ 1992;304:339343.

62 Lebovitz HE, Wiegmann TB, Cnaan A, Shahinfar S, Sica DA, Broadstone V, Schwartz SL, Mengel MC, Segal R, Versaggi JA, et al: Renal protective effects of enalapril in hypertensive NIDDM: role of baseline albuminuria. Kidney Int Suppl 1994;45:S150S155. 